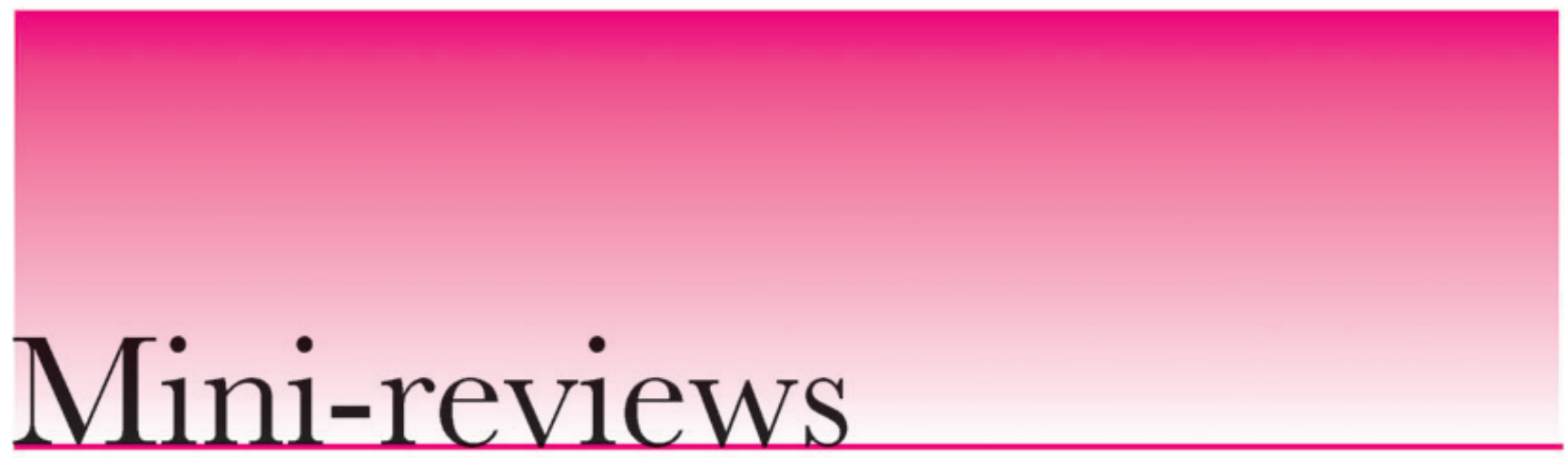

The four mini-reviews are all descriptive of various aspects of prostatic disease. The first three relate to prostate cancer; the molecular staging of prostate cancer is a potential hightechnology way of helping to decide more accurately which is the most appropriate treatment for a given patient. Transition zone cancers are reviewed extensively in two papers, the first dealing with pathological aspects and the second with clinical aspects.

Finally, the three methods of treating chronic prostatitis are reviewed, and suggestions made as to the correct selection of treatment for a particular type of patient.

\title{
The molecular staging of prostate cancer
}

IAIN G. McINTYRE, CLAIRE A. HART, MICHAEL D. BROWN, DAVID G. ROSS, NICHOLAS J.R. GEORGE* and NOEL W. CLARKE

Genito-Urinary Cancer Research Group, Christie Hospital, Manchester, *Department of Urology, University Hospital of South Manchester, Manchester, UK

Accepted for publication 18 April 2004

\section{KEYWORDS}

prostate adenocarcinoma, micrometastasis, staging, RT-PCR

\section{INTRODUCTION}

The natural history of prostate cancer is notoriously variable and whilst some tumours remain indolent for many years, others result in rapid progression to metastasis and death. A substantial minority of patients treated with radical prostatectomy or radiotherapy with curative intent have rising levels of PSA in their serum after treatment, and this is often associated with the eventual development of metastases. It is possible that some of these patients already have 'micrometastases' at the time of treatment. No staging investigation is able to predict reliably which patients will be cured by radical treatment. Radionuclide bone scans are positive in only $5 \%$ of patients with a serum PSA of $<20 \mathrm{ng} / \mathrm{mL}$ [1], although a substantial number of patients with negative bone scans will not be cured. CT and MRI are not reliable for local staging of tumours and newer investigations, e.g. positron emission tomography and immunoscintigraphy, are as yet unable to detect micrometastases. Partin's tables, which use serum PSA, clinical stage and Gleason grade to predict local stage and the likelihood of lymph node involvement, are commonly used to assess the chances of a localized prostatic tumour being cured by radical surgery [2]. These tables depend on the accuracy of clinical staging by a DRE and the histological grade of biopsy material, neither of which is entirely dependable.

During the process of metastasis prostate cancer cells are thought to migrate from the prostate to the bone marrow via the blood stream. Detecting significant numbers of such cells in samples of peripheral venous blood or bone marrow might suggest that a patient is at risk of developing metastases. It could be argued that such a patient would be unlikely to be cured by radical local therapy and that in some circumstances he may benefit from early systemic therapy. The recent development of sensitive molecular detection techniques such as RT-PCR has enabled the detection of very small numbers of prostate cells. To date, many studies have investigated the role of detecting prostate epithelial cells by RT-PCR as a staging technique. These studies are based on the hypothesis that prostate cells are not normally detectable outside the prostate gland and that the presence of a 'critical number' of prostate cells in the blood or bone marrow correlates with eventual disease progression.

\section{RT-PCR}

RT-PCR can be used to detect minute amounts of mRNA that codes for specific proteins such as PSA, and can thus be used to detect very small numbers of cells producing 
these proteins. Total mRNA is first converted to cDNA by the enzyme RT. Using prostatespecific oligonucleotide primers, defined prostate sequences can be amplified by the PCR, which uses the thermostable Taq DNA polymerase. Up to 40 consecutive amplification cycles can be achieved over a 2-h period. RT-PCR was first used to detect prostate cells in lymph nodes and bone marrow of prostate cancer patients in 1992 [3].

\section{REQUIREMENTS FOR A MOLECULAR STAGING TEST}

To be useful as a staging technique, RT-PCR needs to be both specific for prostate cells and sensitive enough to detect very few of them. It should be useable on an easily available clinical sample and would ideally give some idea of the metastatic potential of any prostate cells detected, thereby facilitating prediction of the natural history of the disease.

Prostate specificity: There are several proteins that are only rarely found outside the prostate gland; these include PSA, prostate-specific membrane antigen (PSMA), human kallikrein 2 (hK2), prostate stem cell antigen and DD3 (a noncoding gene whose expression is extremely prostate-specific). The mRNAs for these proteins thus have the potential to be used as circulating cell markers.

Sensitivity: Animal studies have suggested that $\approx 10000$ tumour cells need to be present in the systemic circulation before metastasis is likely to occur [4]. The ability to detect this number of tumour cells, equating to 10 cells/ $5 \mathrm{~mL}$ blood sample, would thus be required of a molecular staging assay.

Clinical material: Peripheral venous blood or iliac crest bone marrow, which can be obtained painlessly under local anaesthesia, can both be used for RT-PCR assays, as can pelvic lymph nodes obtained at open surgery.

\section{RT-PCR ASSAY SENSITIVITY AND SPECIFICITY}

In vitro sensitivity: The sensitivity of RT-PCR assays is established by testing known numbers of cells from prostate cell lines, e.g. LNCaP, diluted in many white blood cells (WBC). Almost all studies published thus far have achieved an assay sensitivity of one $\mathrm{LNCaP}$ cell detectable in $10^{6} \mathrm{WBC}$, with some able to detect one prostate cell in $10^{8}$ background blood cells [5]. Such assays should therefore be able to detect 10 prostate cells in a $5 \mathrm{~mL}$ sample.

Clinical sensitivity: Many published RT-PCR studies have tested blood samples from patients with advanced disease. Most such patients might be expected to have detectable circulating prostate cells, but in fact the percentage of positive RT-PCR results varies significantly among studies, from $42 \%$ [6] to $100 \%$ [7]. It is now known that assay sensitivity can be improved by using more sample replicates in the PCR [8]. This compensates for the 'sampling error' inherent in testing very small volumes (microlitres) of a very dilute solution of DNA.

Specificity: Most RT-PCR studies so far have shown no evidence of prostate cells in the blood or bone marrow of controls without prostate cancer. However, a significant minority of published studies reported significant numbers of positive RT-PCR results in such samples [9], probably because of technical error. False-positive results can be eliminated by using fewer amplification cycles and thus reducing the sensitivity of the RTPCR reaction [8]. The most likely explanation for the false-positive results is that these extremely sensitive RT-PCR assays can detect the tiny amount of prostate-specific RNA that results from background 'illegitimate' or leaky transcription of all genes in all types of dividing cell in the body.

\section{CLINICAL UTILITY OF RT-PCR FOR STAGING}

\section{BLOOD SAMPLES}

Because of the relatively recent advent of RTPCR the length of follow-up of studies published so far is short (rarely over a median of 2 years). Most authors attempting to assess the clinical utility of RT-PCR have compared assay results with pathological results at radical prostatectomy, with some proceeding to assess postoperative biochemical recurrence. In 1994 Katz et al. [5] took blood samples from 319 patients before radical prostatectomy and analysed them with a PSA RT-PCR assay; $16 \%$ of patients with pathological stage pT2 and 51\% with pT3 disease were RT-PCR positive. The same group later showed a correlation with postoperative biochemical recurrence in the same series of patients [10]. However, many other early RTPCR studies failed to show any correlation between RT-PCR results and clinical variables, probably because of differences in the methods. More recent results have generally been better. In 2001 Straub et al. [11] reported a significant correlation between the results of a real-time quantitative RT-PCR assay and pathological stage. Our research group reported a significant relationship between RT-PCR results and PSA progression in patients managed by watchful waiting [12]. A strong correlation has also been established between RT-PCR results after surgery and progressive biochemical failure [13]. The presence of RT-PCR-detectable prostate cells has been shown to be associated with significantly lower survival in patients with advanced prostate cancer [14]. One study to date has shown that the development of metastases after radical surgery (defined as a serum PSA of $>0.1 \mathrm{ng} / \mathrm{mL}$ and visceral or lymph node metastases on CT) is significantly more likely in RT-PCR-positive than -negative patients [15].

\section{BONE MARROW}

Fewer studies have investigated the use of RTPCR in detecting prostate epithelial cells in bone marrow aspirates. In 1994 Wood et al. [16] used PSA-RT-PCR in bone marrow from 43 patients who had a radical prostatectomy and found that $20 \%$ of those with pathologically localized disease were RT-PCRpositive, vs $65 \%$ of those with extraprostatic disease $(P=0.003)$. The same group later reported a significant correlation between the bone marrow PSA-RT-PCR result and both postoperative serum PSA level and pathological stage [17]. In general there have been fewer bone marrow studies, although these tend to show a better correlation with clinical variables than do the blood-based RT-PCR studies.

\section{TECHNICAL ISSUES}

RT-PCR results published so far vary greatly in their sensitivity, specificity and apparent clinical utility. RT-PCR is a complex, multistage molecular technique and most research groups have used their own 'unique' assay, hence the variation in results. Major sources of interstudy variation include the following: 
Amount of RNA sampled: There is great variability among studies in the proportion of the total mRNA isolated from the initial sample that is subjected to reverse transcription, and in the amount of the resultant reverse transcription product (cDNA) that is amplified in the PCR reaction. Katz et al. [5] used 1\% of total sample RNA in each PCR reaction. The use of much more RNA than this to improve assay sensitivity tends to produce false-positive results.

Primers: These are DNA fragments which delineate the ends of the specific sequence under investigation. There is no obvious general difference between primers for PSA, PSMA and hK2 in terms of assay sensitivity. However, many different primers (DNA strands at the ends of the sequence to be amplified) and hence amplified sequences have been used for each molecule. Jung et al. [18] found a 10-fold difference in sensitivity if different primers for PSA were used with otherwise identical reaction conditions. We have found, like others, that using more than one primer, e.g. PSA and PSMA together, can increase assay sensitivity [8].

Detection of amplified DNA: The traditional method for visualizing the DNA amplified by RT-PCR is by adding ethidium bromide, an agent that intercalates the DNA molecule, rendering it visible under ultraviolet light. This method gives a 'yes or no' result for the presence of the target DNA, with little idea of the amount present. Many workers have further enhanced the assay sensitivity by using ${ }^{32}$ P-labelled nucleotides in the PCR and autoradiographing the PCR products, by southern blotting the PCR product, or by using a 'nested primer' technique to further amplify the PCR product DNA before gel electrophoresis.

Quantitative RT-PCR: Although most of the RT-PCR assays published to date give only a 'yes or no' answer for the presence of prostate cells, it is possible to measure the amount of DNA amplified and hence estimate the number of prostate cells present in the original sample. The amount of PCR product DNA can be estimated by using radiolabelled nucleotides in the PCR reaction and quantifying the radioactivity of the product. This only gives an approximate idea of the amount of target DNA in the original sample because of the inherent variability of the PCR amplification process. This amplification variability is eliminated by the relatively new technique of real-time quantitative RT-PCR, in which the amount of fluorescence-labelled PCR product is monitored continuously during amplification. Real-time RT-PCR has been used successfully in detecting prostate epithelial cells [11].

\section{NON-MOLECULAR DETECTION OF SINGLE PROSTATE CELLS}

RT-PCR has several technical problems which impair assay reproducibility. Identifying intact prostate cells, rather than their mRNA, would circumvent some of these technical problems, as well as potentially allowing the study of the malignant potential of any cells identified.

Epithelial cells can be isolated from blood or marrow by density-gradient centrifugation, which removes red blood cells, and by immunocytochemical staining. The ease of detection of the resulting few epithelial cells amongst very many WBC can be improved by an immunomagnetic enrichment process, in which the cellular component of the sample is incubated with microscopic metal beads complexed to an epithelial cell antibody. The cells are then passed through a magnetized column which retains the epithelial cells whilst allowing WBC to pass to through. This process is $\approx 80 \%$ efficient at detecting prostate cells [19]. There are relatively few reports of the successful isolation of prostate cells from blood samples. Brandt et al. [20] found that eight of 10 patients with prostate cancer had a few detectable circulating prostate cells (usually fewer than 15 per $40 \mathrm{~mL}$ blood sample) using an immunomagnetic separation technique. However, several studies have used the technique with success on bone-marrow samples. The presence in bone marrow of prostate cells detected by staining with a panepithelial antibody has been shown to be associated with disease progression [21], and further studies showed that $36 \%$ of prostate cells found in bone marrow are actively proliferating [22]. It is also possible to use immunomagnetic separation of cells in conjunction with RT-PCR to improve the balance between sensitivity and specificity [23].

Small numbers of prostate cells can also be detected by flow cytometry, a technique in which prostate-specific antibodies conjugated to a fluorescent marker are used to mark prostate cells in the sample, and these can then be sorted from background blood cells and counted. This is generally regarded as being a less sensitive detection technique than either RT-PCR or immunocytochemical staining. However, a recent study showed that the presence of many prostate cells detected by flow cytometry was associated with rapid disease progression [24].

\section{CONCLUSION}

Early results of studies of the utility of RT-PCR as a molecular staging technique were very promising, showing a clear correlation between RT-PCR results and both pathological failure of and biochemical recurrence after radical prostate surgery. Early 'follow on' studies, using different RT-PCR methods, were unable to reproduce these results. This variability is almost certainly caused by technical differences in the RT-PCR assays used. Small changes in assay method can result in great differences in the amplification power of RT-PCR. In our laboratory we have found that careful optimization of reaction conditions is essential to achieve a good balance between assay sensitivity and specificity. In turn, such a balance is essential for the assay to be useful clinically. The rapid emergence of new molecular technology, e.g. real-time quantitative RT-PCR, promises to make assay optimization easier.

The results of RT-PCR studies published in recent years are generally more promising than the early results. This reflects recent technical advances and increased experience with this complex molecular assay. Most studies published recently show a significant correlation between peripheral blood RT-PCR results and treatment failure in localized prostate cancer, and those studies using bone marrow for RT-PCR analysis tend to give better clinical correlations than those using venous blood.

None of the RT-PCR studies to date involve many patients and, in such a rapidly developing field, results tend to be published after a minimum follow-up. It is therefore difficult to assess the true prognostic significance of a positive RT-PCR result. The hypothesis upon which RT-PCR studies are based (that significant numbers of detectable prostate cells outside the prostate predict disease progression) has certainly not so far been tested rigorously. 
The constant improvement of molecular technology and the advent of real-time RTPCR hold great promise for the RT-PCR assay as a molecular staging technique, and further study is certainly merited. This would require assay standardization and the recruitment of many patients with a long follow-up. Only when these studies have been undertaken properly will it be possible to assess the true utility of 'molecular staging' in prostate cancer.

\section{CONFLICT OF INTEREST}

None declared.

\section{REFERENCES}

1 Albertsen PC, Hanley JA, Harlan LC et al. The positive yield of imaging studies in the evaluation of men with newly diagnosed prostate cancer: a population based analysis. J Urol 2000; 163: 113843

2 Partin AW, Kattan MW, Subong NP et al. Combination of prostate-specific antigen, clinical stage and Gleason score to predict pathological stage of localised prostate cancer. JAMA 1997; 277: 144551

3 Vessella RL, Riley DE, Blouke $K A$, Arfman EW. A sensitive method for detection of prostate tumour cell marker using the polymerase chain reaction. J Urol 1992; 147: 441A

4 Fidler IJ. Critical factors in the biology of human cancer metastasis: Twenty eighth GHA Clowes Memorial Lecture. Cancer Res 1990; 50: 6130-8

5 Katz AE, Olsson CA, Raffo AJ et al. Molecular staging of prostate cancer with the use of an enhanced reverse transcriptase-PCR assay. Urology 1994; 43: 765-75

6 Gao CL, Maheshwari S, Dean RC et al. Blinded evaluation of reverse transcriptase polymerase chain reaction prostate specific antigen peripheral blood assay for molecular staging of prostate cancer. Urology 1999; 53: 714-21

7 Ignatoff JM, Oefelein MG, Watkin W, Chmiel JS, Kaul KL. Prostate specific antigen reverse transcriptase polymerase chain reaction assay in preoperative staging of prostate cancer. J Urol 1997; 158: $1870-5$
8 McIntyre IG, Spreckley K, Clarke RB, Anderson E, Clarke NW, George NJ. Optimization of the reverse transcriptase polymerase chain reaction for the detection of circulating prostate cells. Br J Cancer 2000; 83: 992-7

9 Henke W, Jung $M$, Jung $K$ et al. Increased analytical sensitivity of RTPCR of PSA mRNA decreases diagnostic specificity of detection of prostate cells in blood. Int J Cancer 1997; 70: 52-6

10 de la Taille A, Olsson CA, Buttyan R et al. Blood-based reverse transcriptase polymerase chain reaction assays for prostatic specific antigen: long term follow-up confirms the potential utility of this assay in identifying patients more likely to have biochemical recurrence (rising PSA) following radical prostatectomy. Int J Cancer 1999; 84: 360-4

11 Straub B, Muller M, Krause $\mathrm{H}$ et al. Detection of prostate-specific antigen RNA before and after radical retropubic prostatectomy and transurethral resection of the prostate using 'LightCycler'-based quantitative real-time polymerase chain reaction. Urology 2001; 58: 815-20

12 McIntyre IG, Clarke RB, Anderson E, Clarke NW, George NJ. Molecular prediction of progression in patients with conservatively managed prostate cancer. Urology 2001; 58: 762-6

13 Tombal B, Cangh PJ, Loric S, Gala JL. Prognostic value of circulating prostate cells in patients with a rising PSA after radical prostatectomy. Prostate 2003; 56: 163-70

14 Kantoff PW, Halabi S, Farmer DA, Hayes DF, Vogelzang NA, Small EJ. Prognostic significance of reverse transcriptase polymerase chain reaction for prostate-specific antigen in men with hormone-refractory prostate cancer. J Clin Oncol 2001; 19: 3025-8

15 Mejean A, Vona G, Nalpas B et al. Detection of circulating prostate derived cells in patients with prostate adenocarcinoma is an independent risk factor for tumour recurrence. J Uro/ 2000; 163: 2022-59

16 Wood DP, Banks ER, Humphreys S, McRoberts JW, Rangnekar VM. Identification of bone marrow micrometastases in patients with prostate cancer. Cancer 1994; 74 : 2533-40
17 Wood DP, Banerjee M. Presence of circulating prostate cells in the bone marrow of patients undergoing radical prostatectomy is predictive of diseasefree survival. J Clin Oncol 1997; 15: 34517

18 Jung $R$, Ahmad-Nejad P, Wimmer M, Gerhard M, Wagener C, Neumaier M. Quality management and influential factors for the detection of single cells by reverse transcriptase polymerase chain reaction. Eur J Chem Clin Biochem 1997; 35: 3-19

19 Meye A, Bilkenroth U, Schmidt U et al. Isolation and enrichment of urologic tumor cells in blood samples by a semi-automated CD45 depletion autoMACS protocol. Int J Oncol 2002; 21: 521-30

20 Brandt B, Junker J, Griwatz C et al. Isolation of prostate derived cells and cell clusters from human peripheral blood. Cancer Res 1996; 56: 4556-61

21 Weckermann D, Muller P, Wawroschek F, Harzmann R, Riethmuller G, Schlimok G. Disseminated cytokeratin positive tumor cells in the bone marrow of patients with prostate cancer: detection and prognostic value. J Urol 2001; 166: 699-703

22 Bianco FJ Jr, Wood DP Jr, Gomes de Oliveira J, Nemeth JA, Beaman AA, Cher ML. Proliferation of prostate cancer cells in the bone marrow predicts recurrence in patients with localized prostate cancer. Prostate 2001; 49: 23542

23 Ghossein RA, Osman I, Bhattacharya $S$ et al. Detection of prostatic specific membrane antigen messenger RNA using immunobead reverse transcriptase polymerase chain reaction. Diagn Mol Pathol 1999; 8: 59-65

24 Moreno JG, O'Hara SM, Gross S et al. Changes in circulating carcinoma cells in patients with metastatic prostate cancer correlate with disease status. Urology 2001; 58: 386-92

Correspondence: Iain G. McIntyre, Consultant Urologist, Department of Urology, Manchester Royal Infirmary, Oxford Road, Manchester M13 9WL, UK. e-mail: iain.mcintyre@cmmc.nhs.uk

Abbreviations: PSMA, prostate-specific membrane antigen; hK2, human kallikrein 2; WBC, white blood cells. 